Document downloaded from:

http://hdl.handle.net/10251/56504

This paper must be cited as:

Perea Rojas Marcos, F.; Puerto Albandoz, J.; Fernández García, FR. (2012). Avoiding unfairness of Owen allocations in linear production processes. European Journal of Operational Research. 220(1):125-131. doi:10.1016/j.ejor.2012.01.013.

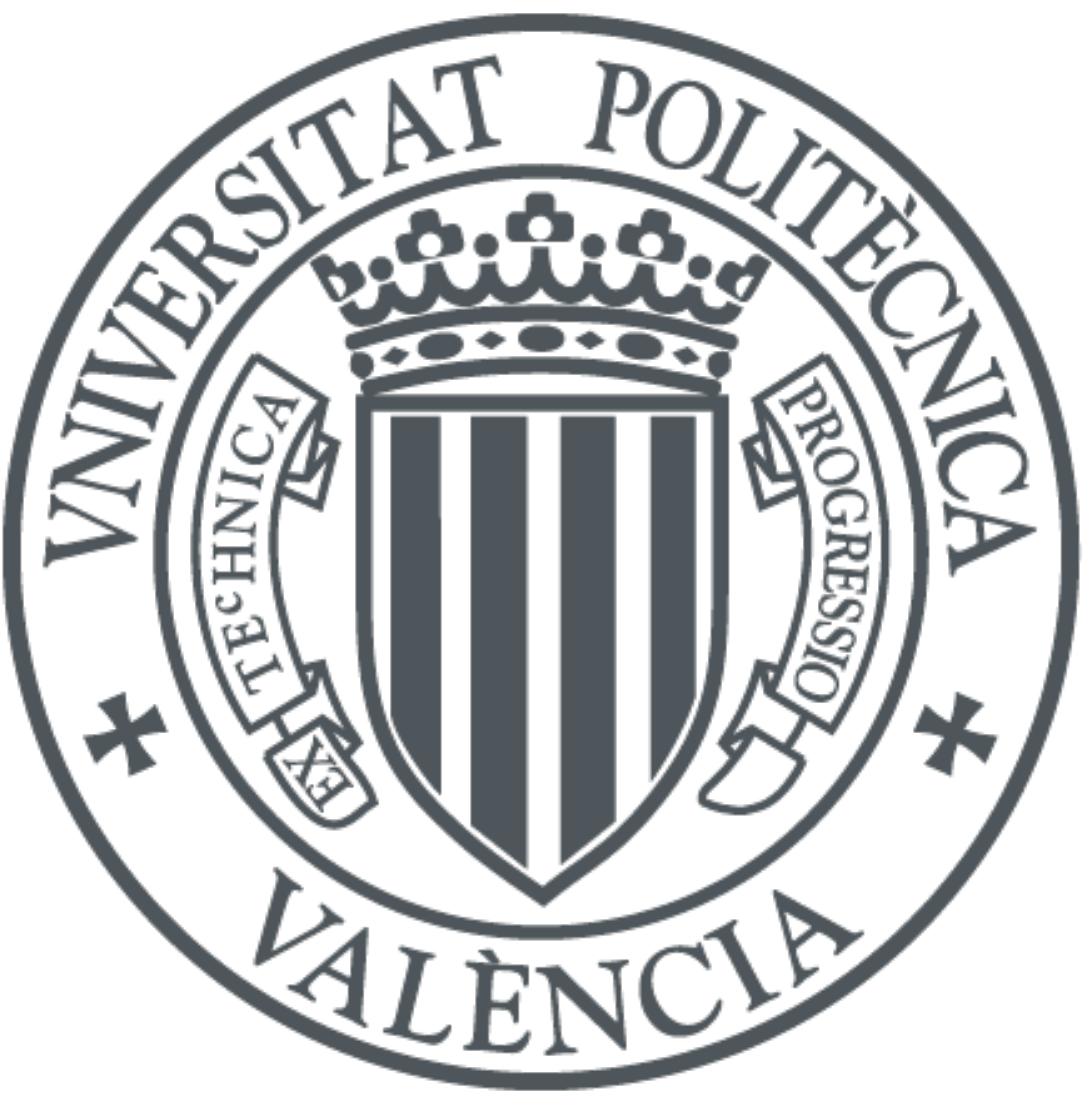

The final publication is available at

http://dx.doi.org/10.1016/j.ejor.2012.01.013

Copyright Elsevier

Additional Information 


\title{
Avoiding unfairness of Owen allocations in linear production processes
}

\author{
Federico Perea ${ }^{1}$ (Corresponding Author), \\ Justo Puerto² ${ }^{2}$ Francisco R. Fernández ${ }^{2}$ \\ email: perea@eio.upv.es,\{puerto,fernande\}@us.es, \\ telephone and fax: +34963877490 / +34 963877499 \\ ${ }^{1}$ Universitat Politècnica de València (Spain) \\ ${ }^{2}$ Universidad de Sevilla (Spain)
}

\begin{abstract}
This paper deals with cooperation situations in linear production problems in which a set of goods are to be produced from a set of resources so that a certain benefit function is maximized, assuming that resources not used in the production plan have no value by themselves. The Owen set is a well-know solution rule for the class of linear production processes. Despite their stability properties, Owen allocations might give null payoff to players that are necessary for optimal production plans. This paper shows that, in general, the aforementioned drawback cannot be avoided allowing only allocations within the core of the cooperative game associated to the original linear production process. In this paper a new solution set named EOwen is introduced. For any player whose resources are needed in at least one optimal production plan, the EOwen set contains at least one allocation that assigns a strictly positive payoff to such player.
\end{abstract}

Keywords: Cooperative games, Linear production games, Allocations. 


\section{Introduction}

A benefit cooperative game is a pair $(N, v)$, where $N=\{1,2, \ldots, n\}$ is the set of players and $v: 2^{N} \rightarrow \mathbb{R}$ is the characteristic function assigning to every coalition $S \subset N$ the maximum benefit that the cooperation between players in $S$ would yield. For a complete introduction on cooperative game theory see for instance Owen (1995) or Forgó et al. (1999). Assuming that the game is superadditive, that is $v(S)+v(T) \leq v(S \cup T), \forall S, T \subset N$, cooperation among all players is beneficial and, therefore, the grand coalition $N$ is to form.

One of the main questions in cooperative game theory is how to distribute the benefit obtained by the grand coalition $N$ among the players. An allocation is a vector $\alpha \in \mathbb{R}^{n}$, such that $\alpha_{i}$ is the payoff of player $i$ and $\sum_{i=1}^{n} \alpha_{i}=v(N)$. One well-accepted way of allocating $v(N)$ among the players is to find allocations in the core. The core of a game $(N, v)$, denoted by $\operatorname{Core}(N, v)$, is the set of allocations satisfying that no coalition of players can obtain a better payoff by acting separately from the rest of players. That is,

$$
\operatorname{Core}(N, v)=\left\{\alpha \in \mathbb{R}^{n}: v(S) \leq \alpha(S) \forall S \subset N, v(N)=\alpha(N)\right\}
$$

where $\alpha(S)=\sum_{i \in S} \alpha_{i}, \forall S \subset N$. In principle, the core has at least two problems: the core of a game might be empty, that is, there are games for which no core allocations exist, and finding a core allocation might be a NPhard problem. Along the years, many other allocation rules have appeared in the literature. One of the most used allocation rules is the Shapley value, 
which has attracted a lot of interest for its many applications, see Moretti and Patrone (2008).

A linear production problem is a situation in which certain goods that can be sold in a market are to be produced from a set of available distinct resources. An implicit feature of the linear production problems we deal with in this paper is that the resources not used in the production plan have no value at all. Situations like this may arise when the resources are perishable and, if not used in the next production plan, they are wasted. Another example of this situation is found in some industries in developed countries that give their excesses to underdeveloped countries, to charity organizations, or even to other companies within the same area as long as they are not competing ones. This is beneficial for both parties: the donor party gets rid of excesses which, if not used, must be eliminated at certain cost, and the receiving party only has to pay for the shipping costs, which is usually cheaper than having to buy the material.

In this paper we study a new set of allocations for linear production processes (LP processes for short), which arise when a bunch of players $N=$ $\{1, \ldots, n\}$ with conflicting objectives control the resources of a LP problem. A cooperative game, called LP game, can be associated to each LP process. (Note that different LP processes may generate the same LP game.) An early reference to LP games can be found in Owen (1975). LP games are totally balanced games, so every subgame of a LP game has a non-empty core. By solving the dual problem of the underlying linear production problem we can obtain a set of allocations for LP processes known as Owen allocations (see Owen (1975)), which has been well-studied in the literature. One of its 
main properties is that Owen allocations are always core allocations, and are easily computed. More recently, Van Gellekom et al. (2000) provided an axiomatic characterization of this solution set. In this paper we show that, despite their stability properties, Owen allocations do not always yield a fair distribution of the benefit obtained. For instance, a player whose resources are necessary for any optimal plan may receive a null payoff from Owen allocations. Such drawback is discussed in this paper, and an alternative allocation set is proposed.

Since the pioneering work by Owen, several generalizations of LP games have appeared in the literature. Dubey and Shapley (1984) study a game in which players have partial control over the constraints of a general mathematical programming problem. Granot (1986) introduces another generalization in which the resources owned by a coalition are not restricted to be the sum of the resources of players in the coalition. Curiel et al. (1989) introduce LP games with committee control, obtaining results on the balancedness of these games, whose core has been more recently studied by Molina and Tejada (2004).

The goal of this paper is to introduce a new set of allocations for linear production processes that avoid some of the aforementioned drawbacks of the Owen set. To this end, the rest of the paper is structured as follows. Section 2 gives a short introduction to LP processes and a motivation of the studied problem. Some definitions and technical results are given in Section 3. The allocation set proposed in this paper is introduced and analyzed in Section 4. An axiomatic characterization and some of its properties are given, as well as a discussion about the impossibility of finding core allocations that avoid 
the unfairness problem of the Owen allocations we address in this paper.

\section{Linear Production processes}

A LP problem is a situation in which there is a finite set of resources $R=\{1,2, \ldots, r\}$ and from those resources a set $P=\{1,2, \ldots, p\}$ of consumption goods can be produced. The production technologies are given by a matrix $A \in \mathbb{R}^{r \times p}$, where $A_{i j} \geq 0$ denotes the amount of resource $i$ necessary to produce one unit of product $j, \forall i=1, \ldots, r, j=1, \ldots, p$. It is also assumed that the demand of every product is large enough to sell all produced products, the unitary market price of product $j$ being $c_{j} \geq 0$. The objective of a LP problem is to decide how much of each product should be produced so that the general benefit is maximized.

Assume now that a group of players $N=\{1, \ldots, n\}$ control the resources $R=\{1,2, \ldots, r\}$, that is, player $k$ owns $B_{i k} \geq 0$ units of resource $i, k=$ $1, \ldots, n, i=1, \ldots, r$. Therefore, let $B=\left(B_{i k}\right)_{r \times n}$ be the resource-player matrix. Let $b \in \mathbb{R}^{r}$ be the resource vector, that is $b=B e_{N}$, where $e_{S} \in \mathbb{R}^{n}$ satisfying $\left(e_{S}\right)_{k}=1$ if $k \in S$, and zero otherwise for all $S \subseteq N$. In other words, $b_{i}$ is the total amount of resource $i$ owned by the grand coalition, that is, $b_{i}=\sum_{k=1}^{n} B_{i k} \forall i \in R$. Thus, the maximum profit that can be made by the cooperation of all players is the value of problem $P_{N}$ :

$$
\begin{aligned}
& \max c x \quad \min y b
\end{aligned}
$$

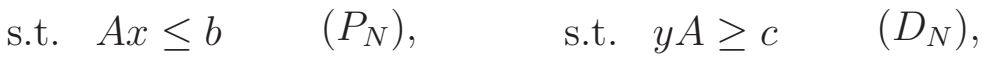

$$
\begin{aligned}
& x \geq 0 \quad y \geq 0
\end{aligned}
$$

where $D_{N}$ is the dual problem of $P_{N}$ (see Bazaraa et al. (1990) for a description of duality theory in linear programming). It is easy to check that, 
although players can try to produce separately, it is always more profitable to join their resources since the benefit they obtain this way is at least as high as the sum of the possible coalitions' profits separately. For a coalition $S \subset N$, we define its characteristic function, $v(S)$, via the optimal value of problem $P_{S}$ :

$$
\begin{aligned}
& \max c x \quad \min y B e_{S} \\
& \text { s.t. } A x \leq B e_{S} \quad\left(P_{S}\right), \quad \text { s.t. } y A \geq c \quad\left(D_{S}\right) \text {, } \\
& x \geq 0 \quad y \geq 0
\end{aligned}
$$

where $D_{S}$ is the dual of $P_{S}$.

Problem $P_{S}$ is feasible and bounded for all possible coalitions if $B e_{S}>0$, $c \geq 0$ and $\forall j: c_{j}>0$ there is at least one resource $i \in R$ with $A_{i j}>0$.

Each triple $(A, B, c)$ satisfying the conditions above will be called in the following, according to Van Gellekom et al. (2000), a linear production process. Let $\mathcal{L}$ denote the class of LP processes. From the definition of the characteristic function $v$ one can associate to each LP process a cooperative game $(N, v)$. The reader may note that the same LP game can originate from different LP processes.

Now a natural question arises: how to divide the profit made by the grand coalition among the players. Let us introduce some notation that will be useful in the rest of the paper.

Let $(A, B, c) \in \mathcal{L}$. The feasible regions of problems $P_{N}$ and $D_{N}$, see (2), are denoted by

$$
\begin{aligned}
& F_{\max }(A, B, c):=\left\{x \in \mathbb{R}_{+}^{p}: A x \leq b\right\} \\
& F_{\min }(A, B, c):=\left\{y \in \mathbb{R}_{+}^{n}: y A \geq c\right\}
\end{aligned}
$$


respectively. The optimal values of problems $P_{N}$ and $D_{N}$ are denoted by

$$
\begin{aligned}
& v_{\max }(A, B, c):=\max \left\{c x: x \in F_{\max }(A, B, c)\right\}, \\
& v_{\min }(A, B, c):=\min \left\{y b: y \in F_{\min }(A, B, c)\right\},
\end{aligned}
$$

respectively, and the set of optimal solutions to $P_{N}$ and $D_{N}$ by

$$
\begin{aligned}
& O_{\max }(A, B, c):=\left\{x \in F_{\max }(A, B, c): c x=v_{\max }(A, B, c)\right\}, \\
& O_{\min }(A, B, c):=\left\{y \in F_{\min }(A, B, c): y b=v_{\min }(A, B, c)\right\} .
\end{aligned}
$$

A solution rule $\varphi$ on $\mathcal{L}$ is a map assigning to every LP process $(A, B, c) \in$ $\mathcal{L}$ a set $\Gamma \subset \mathbb{R}^{n}$ such that $\sum_{i \in N} \gamma_{i}=v_{\max }(A, B, c)$ for all $\gamma \in \Gamma$. Each member of this set is an allocation. A well-known solution rule for cooperative games is the core, see (1). One well-accepted solution rule specific for LP processes is the Owen set, defined from optimal solutions to the dual problem $D_{N}$.

Definition 1. Let $(A, B, c) \in \mathcal{L}$. The Owen set of $(A, B, c)$ is

$$
\operatorname{Owen}(A, B, c):=\left\{y B: y \in O_{\min }(A, B, c)\right\}
$$

Owen (1975) proved that $\operatorname{Owen}(A, B, c) \subseteq \operatorname{Core}(A, B, c)$ for every $(A, B, c) \in$ $\mathcal{L}$. That is, Owen allocations are stable in the sense that no group of players can obtain a better payoff by acting separately. Despite these good properties, they should not be considered as ideal allocations. See the following example.

Example 1. Consider the 3-player game $(A, B, c) \in \mathcal{L}$ where

$$
A=\left(\begin{array}{ll}
1 & 0 \\
1 & 1 \\
0 & 1 \\
1 & 2
\end{array}\right), \quad B=\left(\begin{array}{lll}
1 & 0 & 1 \\
0 & 4 & 0 \\
1 & 0 & 0 \\
0 & 5 & 0
\end{array}\right), \quad c=\left(\begin{array}{l}
1 \\
2
\end{array}\right) .
$$


The corresponding dual problem $D(N)$ is

$$
\begin{array}{cccc}
\min & 2 y_{1}+4 y_{2}+y_{3}+5 y_{4} & \\
\text { s.t. } & y_{1}+y_{2} & +y_{4} & \geq 1 \\
& y_{2}+y_{3} & +2 y_{4} & \geq 2 \\
& \\
& y_{1}, y_{2}, y_{3}, y_{4} \geq 0 . & &
\end{array}
$$

The characteristic function of the associated game is $v(\{i\})=v(\{1,3\})=$ $0 \forall i=1,2,3, v(\{1,2\})=3, v(\{2,3\})=1, v(\{1,2,3\})=4$. It can be checked that $O_{\min }(A, B, c)=\{(1,0,2,0)\}$ and, therefore, Owen $(A, B, c)=$ $\{(1,0,2,0) B\}=\{(3,0,1)\}$.

This allocation is in the core of the game but, is it a "fair" allocation? Note that player 2 receives nothing but, without his resources, the optimal production plan cannot be achieved. So, the Owen allocation gives a null payoff to a player whose resources are necessary for the optimal production plan.

What happened in Example 1 is a general drawback of the Owen set in LP processes. This is a consequence of the complementary slackness theorem, see Bazaraa et al. (1990), which says that if there is some surplus of resource $i$ in an optimal solution $x^{*} \in O_{\max }(A, B, c)$ (meaning $\left.\left(A x^{*}\right)_{i}<b_{i}\right)$, then $y_{i}^{*}=0 \forall y^{*} \in O_{\min }(A, B, c)$. This condition for optimality conveys a simple economic principle: if there is a positive slack in a constrained primal resource, i.e. there are leftovers, then the additional quantities of that resource must have no value (shadow prices are zero). This means that only players owning resources that generate no surplus have the chance of receiving a strictly positive payoff from Owen allocations, which are based on shadow prices. This fact could make players get rid of their surpluses so that the 
corresponding dual variables are not forced to be null, so they have the possibility of receiving a positive reward from allocations obtained from dual solutions.

The following section presents a new solution rule on LP processes that avoids the drawback previously discussed. It is based on the idea that the surpluses of resources should not be taken into account. Therefore players owning leftovers of resources in an optimal production plan are allowed to get rid of them at no cost, nor benefit, and play a new reduced game. A similar approach was used to allocate the benefits obtained in a distribution model, see Perea et al. (2009). Another example of an allocation trying to avoid unfairness can be found in Çiftçi and Tijs (2009), who give some rules for minimum spanning tree games.

\section{Technical results and definitions}

In this section we introduce some technical results and definitions that will be useful for presenting our new solution rule. First, the concept of intermediate matrix will be needed in the rest of the paper. Let $M, M^{1}, M^{2}$ be three matrices in $\mathbb{R}^{r \times n}$. We say that

$M \in\left[M^{1}, M^{2}\right]$ if and only if $M_{i k}^{1} \leq M_{i k} \leq M_{i k}^{2} \forall i=1, \ldots, r, k=1, \ldots, n$.

Let $(A, B, c) \in \mathcal{L}$ and $x^{*} \in O_{\max }(A, B, c)$ one solution to the corresponding problem $P_{N}$. The coordinates of $x^{*}$ define the amount of consumption goods to be produced. Now consider the LP process in which each player $k$ reduces the amount of its resource $i$ so that the total amount of this resource owned by all agents is $\left(A x^{*}\right)_{i}:=\sum_{j=1}^{p} A_{i j} x_{j}^{*}, \forall i=1, \ldots, r$. 
Therefore, for any optimal solution $x^{*}$, let $B_{i k}^{x^{*}}$ be the updated amount of resource $i$ owned by agent $k$, satisfying that $0 \leq B_{i k}^{x^{*}} \leq B_{i k}$ and $\sum_{k=1}^{n} B_{i k}^{x^{*}}=$ $\left(A x^{*}\right)_{i}$, that is, player $k$ gets rid of $B_{i k}-B_{i k}^{x^{*}} \geq 0$ units of resource $i, \forall i=$ $1, \ldots, r, k=1, \ldots, n$. Since there might be (infinitely) many ways of finding matrices satisfying these constraints, we make use of the set constituted by of all of them.

Given a LP process $(A, B, c)$ and $x^{*} \in O_{\max }(A, B, c)$, the set of all possible reduced resource-player matrices of $(A, B, c)$ associated to $x^{*}$ is

$$
\mathcal{B}\left(A, B, x^{*}\right)=\left\{B^{x^{*}} \in[\Theta, B]: \sum_{k=1}^{n} B_{i k}^{x^{*}}=\left(A x^{*}\right)_{i}, \forall i \in R\right\},
$$

where $\Theta$ denotes the matrix with the appropriate dimensions and all entries equal to zero.

Define the vector $b^{x^{*}} \in \mathbb{R}^{r}$, where $b_{i}^{x^{*}}=\left(A x^{*}\right)_{i}, \forall i \in R$. Note that $b_{i}^{x^{*}}=\sum_{k=1}^{n} B_{i k}^{x^{*}}$ for all $B^{x^{*}} \in \mathcal{B}\left(A, B, x^{*}\right)$. So, for every $x^{*} \in O_{\max }(A, B, c)$ and $B^{x^{*}} \in \mathcal{B}\left(A, B, x^{*}\right)$ a new LP process $\left(A, B^{x^{*}}, c\right) \in \mathcal{L}$ is defined. Its corresponding problems $P_{S}\left(B^{x^{*}}\right)$ and $D_{S}\left(B^{x^{*}}\right)$ are:

$$
\begin{aligned}
& \max c x \quad \min y B^{x^{*}} e_{S} \\
& \text { s.t. } A x \leq B^{x^{*}} e_{S} \quad P_{S}\left(B^{x^{*}}\right), \quad \text { s.t. } y A \geq c \quad D_{S}\left(B^{x^{*}}\right) \text {. } \\
& x \geq 0 \quad y \geq 0 .
\end{aligned}
$$

Remark 1. Note that $P_{N}\left(B^{x^{*}}\right)$ and $D_{N}\left(B^{x^{*}}\right)$ only depend on $x^{*}$, and not on the chosen reduced matrix. Therefore, once $x^{*} \in O_{\max }(A, B, c)$ is fixed, both $O_{\max }\left(A, B^{x^{*}}, c\right)$ and $O_{\min }\left(A, B^{x^{*}}, c\right)$ are constant for any $B^{x^{*}} \in \mathcal{B}\left(A, B, x^{*}\right)$.

The above property leads us to the definition of reduced LP process. 
Definition 2. $\left(A, B^{x^{*}}, c\right)$ is a reduced LP process of $(A, B, c)$ associated to $B^{x^{*}}$, for every $x^{*} \in O_{\max }(A, B, c)$ and every $B^{x^{*}} \in \mathcal{B}\left(A, B, x^{*}\right)$.

The next lemma gives some properties on the value of problems $P_{N}$ and $D_{N}$ and their solution sets, needed for the rest of the paper.

Lemma 1. Let $(A, B, c) \in \mathcal{L}$. Then

1. $v_{\max }(A, B, c)=v_{\max }\left(A, B^{x^{*}}, c\right) \forall x^{*} \in O_{\max }(A, B, c), \forall B^{x^{*}} \in \mathcal{B}\left(A, B, x^{*}\right)$.

2. $v_{\min }(A, B, c)=v_{\min }\left(A, B^{x^{*}}, c\right) \forall x^{*} \in O_{\max }(A, B, c), \forall B^{x^{*}} \in \mathcal{B}\left(A, B, x^{*}\right)$.

3.

$$
\begin{aligned}
O_{\max }(A, B, c) & =\bigcup_{x^{*} \in O_{\max }(A, B, c)} \bigcup_{B^{x^{*}} \in \mathcal{B}\left(A, B, x^{*}\right)} O_{\max }\left(A, B^{x^{*}}, c\right) \\
& =\bigcup_{x^{*} \in O_{\max }(A, B, c)} \bigcap_{B^{x^{*}} \in \mathcal{B}\left(A, B, x^{*}\right)} O_{\max }\left(A, B^{x^{*}}, c\right) .
\end{aligned}
$$

4.

$$
\begin{aligned}
O_{\min }(A, B, c) \subseteq & \bigcap_{x^{*} \in O_{\max }(A, B, c)} \bigcup_{B^{x^{*}} \in \mathcal{B}\left(A, B, x^{*}\right)} O_{\min }\left(A, B^{x^{*}}, c\right) \\
& =\bigcap_{x^{*} \in O_{\max }(A, B, c)} \bigcap_{B^{x^{*}} \in \mathcal{B}\left(A, B, x^{*}\right)} O_{\min }\left(A, B^{x^{*}}, c\right) .
\end{aligned}
$$

\section{Proof.}

1. It follows because $x^{*}$ is a solution to $P_{N}\left(B^{x^{*}}\right) \forall x^{*} \in O_{\max }(A, B, c), \forall B^{x^{*}} \in$ $\mathcal{B}\left(A, B, x^{*}\right)$.

2. The result follows from part 1 of Lemma 1 and the strong duality theorem in linear programming $\left(v_{\max }=v_{\min }\right)$.

3. First consider $\widehat{x} \in O_{\max }(A, B, c)$ and $B^{\widehat{x}} \in \mathcal{B}(A, B, \widehat{x})$. Trivially $\widehat{x} \in$ $O_{\max }\left(A, B^{\widehat{x}}, c\right)$. Then

$$
\widehat{x} \in \bigcup_{x^{*} \in O \max }(A, B, c) \bigcup_{B^{x^{*}} \in \mathcal{B}(A, B, \widehat{x})} O_{\max }\left(A, B^{x^{*}}, c\right) .
$$


Now consider $\widehat{x} \in \bigcup_{x^{*} \in O_{\max }(A, B, c)} \bigcup_{B^{x^{*}} \in \mathcal{B}(A, B, \widehat{x})} O_{\max }\left(A, B^{x^{*}}, c\right)$. Then, there exists $x^{*} \in O_{\max }(A, B, c)$ and $B^{x^{*}} \in \mathcal{B}\left(A, B, x^{*}\right)$ such that $\widehat{x} \in O_{\max }\left(A, B^{x^{*}}, c\right)$.

Thus

$$
\left.\begin{array}{l}
A \widehat{x} \leq b^{x^{*}}=A x^{*} \leq b \\
\widehat{x} \geq 0 \\
c \widehat{x}=v_{\max }\left(A, B^{x^{*}}, c\right)=v_{\max }(A, B, c)
\end{array}\right\} \Rightarrow \widehat{x} \in O_{\max }(A, B, c)
$$

Since $O_{\max }\left(A, B^{1}, c\right)=O_{\max }\left(A, B^{2}, c\right)$ for all $B^{1}, B^{2} \in \mathcal{B}(A, B, \widehat{x})$ (see Remark 1), the result is proven joining (12) and (13).

4. Let $\widehat{y} \in O_{\min }(A, B, c)$ and $\widehat{x} \in O_{\max }(A, B, c)$. Applying the complementary slackness theorem and the strong duality theorem in linear programming, we have that $\widehat{y}_{i}=0$ for all $i$ such that $(A \widehat{x})_{i}<b_{i}$, and if $(A \widehat{x})_{i}=b_{i}$ then $b_{i}=b_{i}^{\widehat{x}}$. Therefore

$$
\widehat{y} b=\sum_{i=1}^{n} \widehat{y}_{i} b_{i}=\sum_{i:(A \widehat{x})_{i}=b_{i}} \widehat{y}_{i} b_{i}=\sum_{i:(A \widehat{x})_{i}=b_{i}} \widehat{y}_{i} b_{i}^{\widehat{x}}=\sum_{i=1}^{n} \widehat{y}_{i} b_{i}^{\widehat{x}}=\widehat{y} b^{\widehat{x}} .
$$

Thus, for each $B^{\widehat{x}} \in \mathcal{B}(A, B, \widehat{x})$

$$
\widehat{y} b^{\widehat{x}}=\widehat{y} b=c \widehat{x}=v_{\max }(A, B, c)=v_{\max }\left(A, B^{\widehat{x}}, c\right)=v_{\min }\left(A, B^{\widehat{x}}, c\right) .
$$

Trivially $\widehat{y} \in F_{\min }\left(A, B^{\widehat{x}}, c\right)$, since problems $D_{N}$ and $D_{N}\left(B^{\widehat{x}}\right)$ have the same constraints. Thus, we conclude that $\widehat{y} \in O_{\min }\left(A, B^{\widehat{x}}, c\right)$ and the result follows from Remark 1.

Note that, if $x^{*} \in O_{\max }(A, B, c)$ and $B^{x^{*}} \in \mathcal{B}\left(A, B, x^{*}\right)$, then $x^{*} \in$ $O_{\max }\left(A, B^{\prime}, c\right)$ for all $B^{\prime} \in\left[B^{x^{*}}, B\right]$. The next lemma states that the set of reduced matrices of such $B^{\prime}$ is contained in that of $B$. 
Lemma 2. Let $x^{*} \in O_{\max }(A, B, c)$, and let $B^{\prime} \in\left[B^{x^{*}}, B\right]$ for some $B^{x^{*}} \in$ $\mathcal{B}\left(A, B, x^{*}\right)$. Then $\mathcal{B}\left(A, B^{\prime}, x^{*}\right) \subseteq \mathcal{B}\left(A, B, x^{*}\right)$.

Proof. Under the hypotheses in the statement, let $\widetilde{B} \in \mathcal{B}\left(A, B^{\prime}, x^{*}\right)$. Since $\widetilde{B} \in\left[\Theta, B^{\prime}\right] \subseteq[\Theta, B]$, and using $(10)$, it is easy to see that $\widetilde{B} \in \mathcal{B}\left(A, B, x^{*}\right)$.

\section{The Extended Owen Set}

In this section a new solution rule for the class of LP processes is presented. It is based on the idea of not taking into account the surplus generated by optimal solutions to the primal linear programming problem defining the value of $v(N)$. Therefore, we first have to find out how much the amount of resources can be reduced while the maximum profit is unchanged. The following lemma gives a hint to answer this question and states that, in linear production processes with only one optimal production plan, the minimum amount of resources needed to generate the maximum profit must be bounded from above by $b^{x^{*}}$, where $\left\{x^{*}\right\}=O_{\max }(A, B, c)$.

Lemma 3. Let $(A, B, c) \in \mathcal{L}$ such that $\left\{x^{*}\right\}=O_{\max }(A, B, c)$. Let $b^{x^{*}}=$ $A x^{*}$. Let $b^{\prime} \in \mathbb{R}^{n}$ be such that $b^{\prime} \leq b^{x^{*}}$. Then, the maximum values of problems $P$ and $P^{\prime}$ coincide if and only if $b^{\prime}=b^{x^{*}}$, where $P$ and $P^{\prime}$ are defined by

$$
\begin{aligned}
& \max c x \quad \max c x \\
& \text { s.t. } A x \leq b \quad(P), \quad \text { s.t. } A x \leq b^{\prime} \quad\left(P^{\prime}\right) \text {. } \\
& x \geq 0 \quad x \geq 0
\end{aligned}
$$


Proof. Let $(A, B, c)$ and $b^{\prime}$ be a LP process and a vector satisfying the conditions of the theorem, respectively. By contradiction, assume that there exists $x^{\prime} \geq 0: A x^{\prime} \leq b^{\prime}, c x^{\prime}=c x^{*}$ with $b^{\prime}<b$. One has that $x^{\prime} \neq x^{*}$, since there exists $j$ such that $A_{j} \bullet x^{\prime} \leq b_{j}^{\prime}<b_{j}^{x^{*}}=A_{j} \bullet x^{*}$. Since $b^{\prime} \leq b$, one has that $x^{\prime}$ is an optimal feasible solution to problem $(P)$. Since we had assumed that there was only one solution to problem $(P)$, and $c x^{\prime}=c x^{*}$, the contradiction appears and the result is proven.

Note that, in the more general case with multiple optimal production plans, we have that the vector $b^{\prime}$ of the previous lemma is dominated by all the resource vectors associated to optimal production plans, that is, $b^{\prime} \geq b^{x^{*}} \forall x^{*} \in$ $O_{\max }(A, B, c)$. This can be easily proven adapting the result of Proposition 4.1 in Perea et al. (2009).

Based on this idea, the definition of the EOwen set follows.

Definition 3. Let $(A, B, c) \in \mathcal{L}$. The Extended Owen set of $(A, B, c)$ is the set

$$
\operatorname{EOwen}(A, B, c)=\bigcup_{x^{*} \in O_{\max }(A, B, c)} \bigcup_{B^{x^{*}} \in \mathcal{B}\left(A, B, x^{*}\right)} \operatorname{Owen}\left(A, B^{x^{*}}, c\right)
$$

Remark 2. The reader may note that, although $O_{\min }\left(A, B^{x^{*}}, c\right)$ is independent of the choice of matrix $B^{x^{*}}$, once $x^{*}$ has been chosen (see Remark 1); Owen $\left(A, B^{x^{*}}, c\right)$ does not necessarily have a similar property.

In order to start gaining insights into this new solution rule, we present the following result, which states that the more matrix $B$ is reduced without lowering $B^{x^{*}}$, the larger the Owen set of the corresponding LP process. 
Proposition 1. Let $\widehat{B} \in\left[B^{x^{*}}, B\right]$ for some reduced matrix $B^{x^{*}}$ associated to an optimal production plan $x^{*}$. Then we have that:

$$
\operatorname{Owen}(A, B, c) \subseteq \operatorname{Owen}(A, \widehat{B}, c) \subseteq \operatorname{Owen}\left(A, B^{x^{*}}, c\right)
$$

Proof. Let $\alpha \in \operatorname{Owen}(A, B, c) \Rightarrow \exists \bar{y} \in O_{\min }(A, B, c): \alpha=\bar{y} B$. Let us see that $\bar{y} \in O_{\min }(A, \widehat{B}, c)$.

$$
\begin{aligned}
& \min y b \quad \min y \widehat{b} \\
& \text { s.t. } y A \geq c(D), \quad \text { s.t. } y A \geq c \quad(\widehat{D}) \text {. } \\
& y \geq 0 \quad y \geq 0
\end{aligned}
$$

Since $\bar{y} \in O_{\min }(A, B, c)$, by definition one has that $\bar{y}$ is optimal for problem $D$. Therefore, $\bar{y}$ is feasible for problem $\widehat{D}$. Besides, as we proved in Lemma 1 , part $4, \bar{y} b=\bar{y} \widehat{b}$. Thus $\bar{y} \in O_{\min }(A, \widehat{B}, c)$. Applying again the complementary slackness theorem, one has that:

$$
\alpha_{k}=\sum_{i=1}^{n} \bar{y}_{i} B_{i k}=\sum_{i: \bar{y}_{i} \neq 0} \bar{y}_{i} B_{i k}=\sum_{i: \bar{y}_{i} \neq 0} \bar{y}_{i} \widehat{B}_{i k} \Rightarrow \alpha=\bar{y} \widehat{B} .
$$

Therefore we have proven that $\alpha \in \operatorname{Owen}(A, \widehat{B}, c)$, and as a consequence $\operatorname{Owen}(A, B, c) \subseteq \operatorname{Owen}(A, \widehat{B}, c)$. Owen $(A, \widehat{B}, c) \subseteq \operatorname{Owen}\left(A, B^{x^{*}}, c\right)$ can be proven analogously.

An immediate corollary to the previous result states that the name Extended Owen set is meaningful, as EOwen contains the Owen set.

Corollary 1. Owen $(A, B, c) \subseteq \operatorname{EOwen}(A, B, c)$ for all $(A, B, c) \in \mathcal{L}$.

The following example proves that the inclusions in Proposition 1 and Corollary 1 may be strict. 
Example 2. Take the LP process from Example 1. One can see that $O_{\max }(A, B, c)=$ $\left\{x^{*}=(2,1)\right\}$. Therefore, $B^{x^{*}}$ (in this case unique) and a choice of $\widehat{B}$ are:

$$
B^{x^{*}}=\left(\begin{array}{ccc}
1 & 0 & 1 \\
0 & 3 & 0 \\
1 & 0 & 0 \\
0 & 4 & 0
\end{array}\right), \quad \widehat{B}=\left(\begin{array}{ccc}
1 & 0 & 1 \\
0 & 3 & 0 \\
1 & 0 & 0 \\
0 & 5 & 0
\end{array}\right)
$$

Then, we obtain that problem $D_{N}\left(B^{x^{*}}\right)$ and that corresponding to $\widehat{B}, D_{N}(\widehat{B})$, are, respectively:

$$
\begin{aligned}
& \min 2 y_{1}+3 y_{2}+y_{3}+4 y_{4} \\
& \text { s.t. } y_{1}+y_{2} \quad+y_{4} \geq 1 \\
& y_{2}+y_{3}+2 y_{4} \geq 2 \\
& D_{N}\left(B^{x^{*}}\right), \\
& y_{1}, y_{2}, y_{3}, y_{4} \geq 0 \\
& \min 2 y_{1}+3 y_{2}+y_{3}+5 y_{4} \\
& \text { s.t. } y_{1}+y_{2} \quad+y_{4} \geq 1 \\
& y_{2}+y_{3}+2 y_{4} \geq 2 \\
& D_{N}(\widehat{B}) \text {. }
\end{aligned}
$$

From the solutions to these problems, which are the convex hulls of $\{(1,0,2,0)$, $(0,1,1,0),(0,0,0,1)\}$ and $\{(1,0,2,0),(0,1,1,0)\}$, respectively, one can see that $O$ wen $\left(A, B^{x^{*}}, c\right)$ (which in this example coincides with $\operatorname{EOwen}(A, B, c)$ ) is the convex hull of $\{(3,0,1),(1,3,0),(0,4,0)\}$ and Owen $(A, \widehat{B}, c)$ is the convex hull of $\{(3,0,1),(1,3,0)\}$. This way we prove that the relations described in Proposition 1 are strict. Besides, note that there are allocations in EOwen $(A, B, c)$ that give player 2 a strictly positive payoff, unlike Owen $(A, B, c)$. Note as well that $\operatorname{EOwen}(A, B, c) \subset \operatorname{Core}(A, B, c)$. 
From their definitions, it is easy to prove that for reduced LP processes, as introduced in Definition 2, the EOwen set and the Owen set coincide. Analogously, one can state that in LP processes where all the resources are completely used, the EOwen set coincides with the Owen set.

The following proposition proves that allocations in the EOwen set distribute exactly $v(N)$ among the players.

Proposition 2. Let $(A, B, c) \in \mathcal{L}$ and let $\gamma \in \operatorname{EOwen}(A, B, c)$. Then $\gamma$ is efficient.

Proof. Let $\gamma \in \operatorname{EOwen}(A, B, c)$. Then there exists $x^{*} \in O_{\max }(A, B, c)$, $B^{x^{*}} \in \mathcal{B}\left(A, B, x^{*}\right)$ and $\widehat{y} \in O_{\min }\left(A, B^{x^{*}}, c\right)$ such that $\gamma_{k}=\sum_{i=1}^{r} \widehat{y}_{i} B_{i k}^{x^{*}} \forall k=$ $1, \ldots, n$. Therefore

$$
\gamma(N)=\sum_{k=1}^{n} \gamma_{k}=\sum_{k=1}^{n} \sum_{i=1}^{r} \widehat{y}_{i} B_{i k}^{x^{*}}=\sum_{i=1}^{r} \widehat{y}_{i} \sum_{k=1}^{n} B_{i k}^{x^{*}}=\sum_{i=1}^{r} \widehat{y}_{i} b_{i}^{x^{*}}=\widehat{y} b^{x^{*}} .
$$

Since $\widehat{y} \in O_{\min }\left(A, B^{x^{*}}, c\right)$ and $x^{*} \in O_{\max }(A, B, c)$, we know that $\widehat{y} b^{x^{*}}=c x^{*}=$ $v(N)$. This concludes that $\gamma(N)=v(N)$.

Another interesting property states that, for all players whose resources are necessary to produce the maximum benefit $v(N)$ in some optimal production plan, there exists an allocation in EOwen that assigns them a strictly positive payoff.

Theorem 1. Let $(A, B, c) \in \mathcal{L}$ and $x^{*} \in O_{\max }(A, B, c)$, and let $k \in N$ be a player such that some of the resources that he owns are needed for the optimal production plan $x^{*}$ to be developed. Then there exists $\alpha \in \operatorname{EOwen}(A, B, c)$ such that $\alpha_{k}>0$. 
Proof. Let $x^{*} \in O_{\max }(A, B, c)$, and $B^{x^{*}} \in \mathcal{B}\left(A, B, x^{*}\right)$. By the strict complementary slackness theorem, if the slack in the $i^{\text {th }}$ constraint of problem $P_{N}\left(B^{x^{*}}\right)$ is zero, then there exists a solution $y$ to $D_{N}\left(B^{x^{*}}\right)$ such that $y_{i}>0$ (see Theorem 10.7 in Vanderbei (1997)). Assuming that the units of the $i^{\text {th }}$ resource owned by player $k$ are needed for the optimal production plan $x^{*}$, it is easy to see that $B_{i k}^{x^{*}}>0$. Then, the payoff of player $k$ from the EOwen allocation $\alpha_{B^{x^{*}}}=y B^{x^{*}}$ is, at least, $y_{i} B_{i k}^{x^{*}}>0$.

Note that this proposition allows us to state that the EOwen set always overcomes the unfairness problem illustrated in Example 1.

Let us now introduce the property of upper limit inclusion (ULI), which will be useful for our characterization of EOwen.

Property 1 (ULI). A solution rule $\varphi$ satisfies ULI if for every $(A, B, c) \in$ $\mathcal{L}$, every $x^{*} \in O_{\max }(A, B, c)$, and every matrix $B^{\prime}$ such that $B^{\prime} \in\left[B^{x^{*}}, B\right]$ for every $B^{x^{*}} \in \mathcal{B}\left(A, B, x^{*}\right)$, we have that $\varphi\left(A, B^{\prime}, c\right) \subseteq \varphi(A, B, c)$.

The following result proves that EOwen satisfies this property.

Proposition 3. EOwen satisfies ULI.

Proof. Let $(A, B, c) \in \mathcal{L}$. Consider $x^{*} \in O_{\max }(A, B, c)$, and let $B^{\prime} \in$ $\left[B^{x^{*}}, B\right]$ for every $B^{x^{*}}$ reduced matrix associated to $x^{*}$. Similarly as we proved in Proposition 1, it can be seen that $O_{\max }\left(A, B^{\prime}, c\right) \subseteq O_{\max }(A, B, c)$. Besides, since we proved in Lemma 2 that $\mathcal{B}\left(A, B, x^{*}\right) \supseteq \mathcal{B}\left(A, B^{\prime}, x^{*}\right)$, we 
have

$$
\begin{aligned}
\operatorname{EOwen}(A, B, c) & =\bigcup_{x^{*} \in O_{\max }(A, B, c)} \bigcup_{B^{x^{*} \in \mathcal{B}\left(A, B, x^{*}\right)}} \operatorname{Owen}\left(A, B^{x^{*}}, c\right) \\
& \supseteq \bigcup_{x^{*} \in O_{\max }\left(A, B^{\prime}, c\right)} \bigcup_{B^{x^{*} \in \mathcal{B}\left(A, B, x^{*}\right)}} \operatorname{Owen}\left(A, B^{x^{*}}, c\right) \\
& \supseteq \bigcup_{x^{*} \in O_{\max }\left(A, B^{\prime}, c\right)} \bigcup_{B^{x^{*} \in \mathcal{B}\left(A, B^{\prime}, x^{*}\right)}} \operatorname{Owen}\left(A, B^{x^{*}}, c\right) \\
& =\operatorname{EOwen}\left(A, B^{\prime}, c\right) .
\end{aligned}
$$

Now we are ready to give a characterization of the EOwen solution rule for linear production processes.

Theorem 2. Let $\varphi$ be a solution rule over $\mathcal{L} . \varphi$ satisfies ULI, coincides with the Owen set over LP processes without leftovers and is minimal if and only if $\varphi \equiv$ EOwen.

\section{Proof.}

- Clearly EOwen coincides with the Owen set in LP processes without leftovers, and as proven in Proposition 3, EOwen satisfies ULI. Let us see that EOwen is minimal. For this purpose, let $\varphi$ be a solution set that coincides with the Owen set in LP processes without leftovers and satisfies ULI, and let $(A, B, c) \in \mathcal{L}$. Therefore, Owen $\left(A, B^{x^{*}}, c\right)=$ $\varphi\left(A, B^{x^{*}}, c\right) \subseteq \varphi(A, B, c)$ for all $x^{*} \in O_{\max }(A, B, c)$ and all $B^{x^{*}} \in$ $\mathcal{B}\left(A, B, x^{*}\right)$. Hence

$$
\begin{aligned}
\operatorname{EOwen}(A, B, c) & =\bigcup_{x^{*} \in O_{\max }(A, B, c)} \bigcup_{B^{x^{*} \in \mathcal{B}\left(A, B, x^{*}\right)}} \operatorname{Owen}\left(A, B^{x^{*}}, c\right) \\
& =\bigcup_{x^{*} \in O_{\max }(A, B, c)} \bigcup_{B^{x^{*} \in \mathcal{B}\left(A, B, x^{*}\right)}} \varphi\left(A, B^{x^{*}}, c\right) \\
& \subseteq \varphi(A, B, c)
\end{aligned}
$$

which proves that EOwen is minimal. 
- Let $\varphi$ be a solution rule over $\mathcal{L}$ satisfying the hypotheses of the theorem. Therefore $\varphi\left(A, B^{x^{*}}, c\right)=O$ wen $\left(A, B^{x^{*}}, c\right) \forall x^{*} \in O_{\max }(A, B, c)$ and for every $B^{x^{*}} \in \mathcal{B}\left(A, B, x^{*}\right)$, because $\left(A, B^{x^{*}}, c\right)$ has no leftovers. Now, since $\varphi$ satisfies ULI we have that $\varphi\left(A, B^{x^{*}}, c\right) \subseteq \varphi(A, B, c)$, therefore

$$
\operatorname{EOwen}(A, B, c)=\bigcup_{x^{*} \in O \max }(A, B, c) \bigcup_{B^{x^{*}} \in \mathcal{B}\left(A, B, x^{*}\right)} \varphi\left(A, B^{x^{*}}, c\right) \subseteq \varphi(A, B, c)
$$

From the minimality of $\varphi$, and since EOwen satisfies the hypotheses of the theorem, $\varphi(A, B, c) \subseteq \operatorname{EOwen}(A, B, c)$. Thus, EOwen $(A, B, c)=$ $\varphi(A, B, c)$.

Let us now introduce the following increasing monotonicity (IM) property that will lead us to another characterization of EOwen set:

Property 2 (IM). A solution rule $\varphi$ satisfies IM if for every $(A, B, c) \in \mathcal{L}$, every $x^{*} \in O_{\max }(A, B, c)$, and every $B^{1}, B^{2}$ such that $B^{1} \in\left[B^{x^{*}}, B\right]$ for each $B^{x^{*}} \in \mathcal{B}\left(A, B, x^{*}\right)$ and $B^{2} \in\left[B^{1}, B\right]$, we have that $\varphi\left(A, B^{1}, c\right) \subseteq \varphi\left(A, B^{2}, c\right)$.

The following result states that increasing monotonicity is equivalent to upper limit inclusion.

Lemma 4. Let $\varphi$ be a solution rule over $\mathcal{L}$. Then $\varphi$ satisfies ULI if and only if $\varphi$ satisfies $I M$.

Proof. Trivially, if $\varphi$ satisfies IM then it satisfies ULI too. Conversely, let $(A, B, c) \in \mathcal{L}$, and $x^{*} \in O_{\max }(A, B, c)$. Moreover, let $B^{1} \in\left[B^{x^{*}}, B\right]$ for each $B^{x^{*}} \in \mathcal{B}\left(A, B, x^{*}\right)$ and $B^{2} \in\left[B^{1}, B\right]$. It is straightforward that 
$x^{*} \in O_{\max }\left(A, B^{2}, c\right)$. Then, since $B^{1} \in\left[B^{x^{*}}, B^{2}\right]$ and $\varphi$ satisfies IM, we have that $\varphi\left(A, B^{1}, c\right) \subseteq \varphi\left(A, B^{2}, c\right)$, which concludes the proof.

From the equivalency between increasing monotonicity and upper limit inclusion, the following alternative characterization of EOwen trivially follows from Theorem 2 .

Corollary 2. Let $\varphi$ be a solution rule over $\mathcal{L} . \varphi$ satisfies IM, coincides with the Owen set over LP processes without leftovers and is minimal if and only if $\varphi \equiv$ EOwen.

We finish this section by studying the relation between EOwen, the Owen set, and the core of the original game. It is obvious that $\operatorname{Core}(A, B, c) \subseteq$ $\operatorname{Core}\left(A, B^{x^{*}}, c\right)$ for every optimal production plan $x^{*}$ and every reduced matrix $B^{x^{*}}$ associated to it, since $v^{B^{x^{*}}}(S) \leq v(S)$ and $v^{B^{x^{*}}}(N)=v(N)$, where $v^{B^{x^{*}}}$ denotes the characteristic function of the corresponding reduced game. The following example shows that this relation may be strict.

Example 3. Consider the LP process $(A, B, c)$ where

$$
A=\left(\begin{array}{ll}
1 & 0 \\
1 & 1 \\
0 & 1
\end{array}\right), \quad B=\left(\begin{array}{ll}
1 & 0 \\
1 & 3 \\
1 & 0
\end{array}\right), \quad c=\left(\begin{array}{l}
2 \\
1
\end{array}\right)
$$

It can be seen that $O_{\max }(A, B, c)=\left\{x^{*}=(1,1)\right\}$, and the characteristic function of the associated game is $v(\{1\})=2, v(\{2\})=0, v(\{1,2\})=3$. There is a surplus of resource 2. One reduced matrix (in which both players 
drop half of the units of resource 2 they had) is

$$
B^{x^{*}}=\left(\begin{array}{cc}
1 & 0 \\
0.5 & 1.5 \\
1 & 0
\end{array}\right)
$$

and $v^{x^{*}}(\{1\})=1, v^{x^{*}}(\{2\})=0, v(\{1,2\})=3$. Therefore, Core $(A, B, c) \varsubsetneqq$ $\operatorname{Core}\left(A, B^{x^{*}}, c\right)$.

Unfortunately, not all EOwen allocations are core allocations. However, it is not always possible to find allocations that avoid the unfairness drawback of the Owen set mentioned in this paper and remain in the core of the original game at the same time. Therefore, one has to look for some compromise between null-payoff to absolutely necessary players and un-stability. The following example illustrates the above statements.

Example 4. Consider the LP process $(A, B, c)$ with the following data,

$$
A=\left(\begin{array}{ll}
1 & 0 \\
1 & 1 \\
0 & 1
\end{array}\right), \quad B=\left(\begin{array}{lll}
1 & 0 & 0 \\
0 & 1 & 0 \\
0 & 0 & 1
\end{array}\right), \quad c=\left(\begin{array}{l}
1 \\
1
\end{array}\right)
$$

The characteristic function of the associated game is $v(\{1,2\})=v(\{2,3\})=$ $v(\{1,2,3\})=1$, and zero for any other coalition. Therefore, the core of this game consists of the singleton $\{(0,1,0)\}$. Note as well that without the resources of players 1 and 3, player 2 gets nothing, but it is not possible to give a positive payoff to players 1 and 3 with a core allocations.

Let us calculate the EOwen set for this example. One can see that the extreme optimal production plans are $x^{1}=(1,0)$ and $x^{2}=(0,1)$, and that 
$\mathcal{B}\left(A, B, x^{1}\right)$ and $\mathcal{B}\left(A, B, x^{2}\right)$ consist only of one matrix each (named $B^{1}$ and $B^{2}$, respectively). $B^{1}$ has a diagonal equal to $(1,1,0)$ and $B^{2}$ has a diagonal equal to $(0,1,1)$. All non-diagonal entries are null for both matrices. It is easy to see that $O_{\min }\left(A, B^{1}, c\right)$ is the union over $t_{1} \geq 1$ and $t_{2} \geq 0$ of the convex hulls of $\left\{\left(1,0, t_{1}\right),\left(0,1, t_{2}\right)\right\}$, and that $O_{\min }\left(A, B^{2}, c\right)$ is the union over $t_{1} \geq 1$ and $t_{2} \geq 0$ of the convex hulls of $\left\{\left(t_{1}, 0,1\right),\left(t_{2}, 1,0\right)\right\}$. Therefore, Owen $\left(A, B^{1}, c\right)$ is the convex hull of $\{(1,0,0),(0,1,0)\}$ and Owen $\left(A, B^{2}, c\right)$ is the convex hull of $\{(0,0,1),(0,1,0)\}$. For every non-extreme optimal solution $x^{a}=(a, 1-a)(a \in(0,1)), \mathcal{B}\left(A, B, x^{a}\right)$ consists only of one matrix $B^{a}$, in which the diagonal is $(a, 1,1-a)$, and the rest is zero. The corresponding $O_{\min }\left(A, B^{a}, c\right)$ is the convex hull of $\{(0,1,0),(1,0,1)\}$, and therefore the Owen set of the corresponding reduced LP process is $\{(0,1,0),(a, 0,1-a)\}$. Therefore, EOwen $(A, B, c)$ is the convex hull of $\{(1,0,0),(0,1,0),(0,0,1)\}$ (all players can obtain a positive payoff from allocations in this set).

To summarize, the Owen set, the EOwen set and the core of the original game have a relationship as shown in Figure 1.

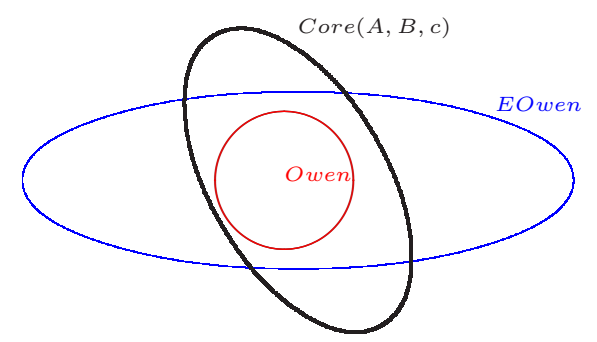

Figure 1: General relation between EOwen, Core and Owen . 


\section{Conclusions}

In this work we have introduced the EOwen set, a new solution rule on the class of linear production processes which overcomes certain drawbacks of the well-known Owen set, in the sense that one can always find an allocation that gives a strictly positive payoff to players whose resources are needed for (at least) one optimal production plan. Some examples in the paper show that Owen allocations do not satisfy this property.

EOwen is defined as the union, over all possible optimal production plans and all possible reduced matrices, of the Owen sets over the corresponding reduced LP processes, in which players get rid of the leftovers in their resources according to a reduced matrix. Several theoretical properties and an axiomatic characterization of this new solution rule are given. By means of an example we also prove that, in general, it is not possible to find allocations that give non-null payoffs to players that are necessary in order to achieve the optimal value of $v(N)$ by restricting to the core.

We note that two types of players have been involved in this paper: group T1, consisting of players such that some of the resources they own are needed for (at least) one optimal production plan; and group T2, consisting of players whose resources are never completely used in any of the optimal production plans. Theorem 1 ensures that players in T1 can always find an allocation in EOwen that gives them a strictly positive payoff. Examples have shown that players in T2 may receive only zero payoffs from Owen allocations. Players in both $\mathrm{T} 1$ and $\mathrm{T} 2$ are of special interest, since they receive strictly positive payoffs from EOwen allocations, and may only receive zero payoffs from Owen allocations (see for instance examples 1 and 2). 
Further research on this topic will focus on methods to find allocations with particular extra properties in the EOwen set, as well as efficient algorithms for obtaining them.

\section{Acknowledgments}

The authors want to thank the Spanish Ministry of Science and Technology for providing financial support under grants MTM200767433 and MTM201019576, and the Junta de Andalucía/Feder (Spain) under grant FQM5849. Special thanks are due to two anonymous referees for their valuable comments and suggestions.

\section{References}

Bazaraa, M., Jarvis, J., Sherali, H., 1990. Linear programming and network flows. Wiley.

Curiel, I., Derks, J., Tijs, S., 1989. On balanced games and games with committee control. OR Spektrum 11 (2), 83-88.

Dubey, P., Shapley, L. S., 1984. Totally balanced games arising from controlled programming problems. Mathematical Programming 29 (3), 245267.

Forgó, F., Szép, J., Szidarovsky, F., 1999. Introduction to the Theory of Games. Kluwer Academic Publishers.

Granot, D., 1986. A generalized linear production model: A unifying model. Mathematical Programming 34 (2), 212-222. 
Çiftçi, B., Tijs, S., 2009. A vertex oriented approach to the equal remaining obligation rules for minimum cost spanning tree situations. TOP 17 (2), 440-453.

Molina, E., Tejada, J., 2004. Linear production games with committee control: Limiting behaviour of the core. European Journal of Operational Research 154 (1), 609-625.

Moretti, S., Patrone, F., 2008. Transversality of the Shapley value. TOP $16(1), 1-41$.

Owen, G., 1975. On the core of linear production games. Mathematical Programming 9 (1), 358-370.

Owen, G., 1995. Game Theory. Academic Press, San Diego.

Perea, F., Puerto, J., Fernández, F. R., 2009. Modeling cooperation on a class of distribution problems. European Journal of Operational Research 198 (3), 726-733.

Van Gellekom, J., Potters, J., Reijnierse, J., Engel, M., Tijs, S., 2000. Characterization of the Owen Set of Linear Production Processes. Games and Economic Behavior 32 (1), 139-156.

Vanderbei, R., 1997. Linear Programming: Foundation and Extensions, 2nd Edition. Kluwer Academic Publisher. 